\title{
The effects of target size and retinal location on a partial-report task of iconic memory
}

\author{
STEVEN C. KLING and GERALD M. LONG \\ Villanova University, Villanova, Pennsylvania
}

\begin{abstract}
Observers are run on a partial-report task under three conditions of background luminance and with two target sizes. Small letters are presented in a small circular array, and large letters are presented in a large circular array. This manipulation is intended to keep letter legibility roughly equivalent as retinal eccentricity varies. At the photopic background level, there is no difference in the decay curves for the two target conditions; but at the mesopic and scotopic background levels, the large-size condition produces greater persistence. An explanation is offered in terms of differential contribution from the cone and rod systems.
\end{abstract}

Within the current literature, there is extensive debate concerning the exact nature, locus, and function of iconic memory, or visual persistence (cf. Bowling \& Lovegrove, 1982, Coltheart, 1980, Haber, 1983, and Long, 1980, 1982). Undoubtedly, an important contributing factor to this unsettled state of affairs has been the numerous empirical discrepancies reported across studies in the area. For example, visual persistence has been found to be positively related to target duration and target luminance in some studies but negatively related to these same variables in other studies (cf. Bowen, Pola, \& Matin, 1974; Sakitt \& Long, 1979a). In the last few years, an increasingly popular way to accommodate such discrepancies has been to suggest that more than a single phenomenon has been assessed in different persistence studies and that the various persistence tasks have inadvertently involved different "kinds" of visual persistence. For example, Phillips (1974) proposed a distinction between "sensory storage" and "schematic visual memory"; Hawkins and Shulman (1979) described "Type I" versus "Type II" visual persistence; and Coltheart (1980) suggested "visible persistence" as distinct from "iconic memory."

A second source of difficulty in the current literature concerns the often overlooked effects of different stimulus values that have been employed across studies. This point is especially important given recent proposals that iconic memory (or visual persistence) may represent the phenomenal consequence of temporal processing limitations of structures very early in the visual system. In this view, an important contribution to performance on many persistence tasks results from the sluggish functioning of the photoreceptors themselves (e.g., Sakitt, 1976; Sakitt \& Long, 1978, 1979a, 1979b). That is, at least one major component to visual persistence is attributed to the time required by the cones and rods to

The authors' mailing address is: Department of Psychology, Villanova University, Villanova, Pennsylvania 19085. return to baseline resting potential following a brief target presentation (e.g., Whitten \& Brown, 1973a, $1973 b, 1973 c)$. In the present context this model of persistence is important because of the myriad of stimulus factors that are known to alter receptor functioning and, by extension, visual persistence as well. Several studies have presented results consistent with this model (Long \& Sakitt, 1980a, 1980b; Sakitt \& Long, 1978, 1979a, 1979b).

In several respects, the present work represents an extension of this theoretical rationale. Changing stimulus conditions from one study to the next are believed to have produced very different results (cf. Adelson \& Jonides, 1980, and Long \& Beaton, 1982). Moreover, the different results across studies (and therefore across stimulus conditions) are believed to reflect predictable interactions among the many retinal factors involved in such stimulus presentations. The present work seeks to demonstrate this point. Stimulus conditions can and do interact, and the results of one stimulus manipulation must be looked at in terms of other stimulus levels. It is believed that the resulting patterns of effects will reveal that very early sensory structures do indeed make a major contribution to the duration of visual persistence.

The stimulus variables of interest in the present work were the retinal location of the target stimulus, the background level under which the target stimulus was viewed, and the acuity demands of the target stimulus. It was believed that these parameters had been too frequently overlooked in their contributions to visual persistence. In examining these particular variables, the present study utilized the version of the partial-report task used by Adelson \& Jonides (1980). Specifically, in such a task, a comparison is made between target arrays that fall on an area of the retina close to the fovea, where the cones are most densely located, and an area of the parafoveal retina where the rod system can begin to contribute to performance. However, a problem with this type of manipulation is that acuity is reduced as 
constant-size targets are presented farther from the fovea. As Eriksen and Schultz $(1977,1978)$ pointed out, the result of peripheral presentation is a degradation in the quality of the stimulus, which in the present study might have led to a processing-rate difference for the target stimuli at the two retinal locations. To avoid this possible confound and to ensure that the target arrays were processed equally at both retinal locations, the stimuli in the present experiment were constructed so as to keep target resolution at, or above, recognition threshold at both retinal locations. This was accomplished by increasing target size as eccentricity increased in order to exceed the recognition limits for letters that have been described in detail by Anstis (1974) at a series of retinal locations. Thus, any processing-rate differences as retinal location was varied were significantly attenuated.

\section{METHOD}

\section{Observers}

Sixty undergraduate students enrolled in general psychology courses at Villanova University served as observers for partial fulfillment of a research requirement for the course. The observers participated individually in a session lasting approximately $90 \mathrm{~min}$. If an observer normally wore spectacles or contact lenses, he/she did so during the experimental session.

\section{Apparatus and Stimuli}

Stimuli were presented using a Gerbrands three-channel tachistoscope (Model G 1132). These stimuli consisted of 32 different eight-letter (uppercase) arrays of black letters (Chartpak Helvetica Bold Extended No. 214). Two different sizes of letters were used for the elements within the target arrays: $.61 \mathrm{deg}$ (8.5 $\mathrm{mm}$ in height and width) or $1.1 \mathrm{deg}(16 \mathrm{~mm}$ in height and width). These letters wer 2 arranged around the circumference of an imaginary circle, the diameter of which varied such that the entire array subtended approximately either 2.0 or $3.6 \mathrm{deg}$. The sizes of the individual elements were $.61 \mathrm{deg}$ within the 2.0-deg array and $1.1 \mathrm{deg}$ within the 3.6-deg array. In other words, as array diameter increased, so did the size of the individual elements within the array. To keep target resolution above threshold at both retinal locations, these values were chosen from the guidelines established by Anstis (1974). Sixteen stimulus cards were constructed for each array size. For these cards, all the letters of the alphabet, except the letters I and Q, were selected randomly, without replacement. The background field contained a small black central fixation point $(.2 \mathrm{deg})$. Additionally, the experiment was conducted in a room with very dim lighting for the duration of the experiment.

\section{Procedure}

The duration of the target stimuli was kept constant at $50 \mathrm{msec}$. The luminance of the target field remained constant at $1.0 \mathrm{fL}\left(3.43 \mathrm{~cd} / \mathrm{m}^{2}\right)$ for the scotopic background condition $(0.01 \mathrm{fL}), 5.0 \mathrm{fL}\left(17.2 \mathrm{~cd} / \mathrm{m}^{2}\right)$ for the mesopic background condition $(1.0 \mathrm{fL})$, and $10.0 \mathrm{fL}\left(34.3 \mathrm{~cd} / \mathrm{m}^{2}\right)$ for the photopic background condition (10.0 fL). The different target luminances were determined in pilot work in order to maintain roughly equivalent persistences at each background level.

For each different interstimulus-interval (ISI) condition $(0,250$, and $500 \mathrm{msec})$, the observers received a block of 16 trials. In these blocks, each of the eight possible positions in the target array was probed twice in a random sequence, with the single constraint that the same position was not probed twice in a row. Each observer's performance was assessed at one array size under the three different ISI values. During a typical experimental session, an observer received three blocks of 16 trials presented in a random order. The observers were assigned randomly to a particular background level and to either the smallor large-target-size condition.

Following a verbal "ready" signal on each trial, the target array replaced the background field for $50 \mathrm{msec}$. After a variable ISI, a probe marker appeared for $20 \mathrm{msec}$ and pointed to one of the positions previously occupied by one of the letters. (The probe marker measured $0.14-$ or 0.29 -deg stroke width, depending on array size.) The observer was instructed to indicate verbally which letter had previously occupied that position, and the experimenter recorded the response without giving the observer any feedback whatsoever. After each block of 16 trials, there was a brief rest period.

Prior to the experimental trials, each observer dark-adapted the left eye for a minimum of $30 \mathrm{~min}$ by wearing two eyepatches (an inner and an outer). During this 30-min adaptation period, viewing was done monocularly with the right eye for several practice trials, and each observer was shown a different random sample of stimulus presentations to orient them to the task. After these practice trials, the observer switched the eyepatches to the right eye, and all experimental trials were done with the observer viewing the stimuli with the dark-adapted left eye.

\section{RESULTS AND DISCUSSION}

For each observer, his/her percentage correct for the 16 trials run at each of the ISI levels is multiplied by eight to obtain an estimate of letters theoretically available per trial. The mean estimates for the $20 \mathrm{ob}$ servers examined under the photopic background level are shown in Figure 1a. These data have been subjected to a 2 (target size) $\times 3$ (ISI) mixed analysis of variance (ANOVA) with repeated measures on the factor of ISI. A significant main effect of ISI is obtained $[F(2,2)=$ $193.89, \mathrm{p}<.001]$. This indicates that visual persistence (iconic memory) is indeed assessed in this experiment and that these results, which reveal a gradual decline in performance with increasing ISI, are consistent with previous research using this general methodology (e.g., Adelson \& Jonides, 1980; Sperling, 1960).

Concerning the other effects depicted in Figure 1, neither the main effect of target size $\left[\mathrm{F}^{\prime}(1,17)<1.0\right]$ nor the ISI $x$ target size interaction $[\mathrm{F}(2,36)<1.0]$ is significant. This indicates that, at least with the photopic background level used with these observers, the combined manipulation of array size and target eccentricity has no effect on partial-report performance. It is argued that this result is consistent with predictions from a retinal model of visual persistence. Such a model would predict little or no differences in partial-report performance as a function of increasing target size, provided that roughly equal discriminability of the targets by the cone system is maintained. Recall that the Anstis (1974) specifications for letter size that were utilized in this experiment were incorporated to keep the targets well above resolution threshold. This was done to avoid any processing-rate differences resulting from degradation of the targets as retinal eccentricity increased. Additionally, at the photopic background level, the rod system's 

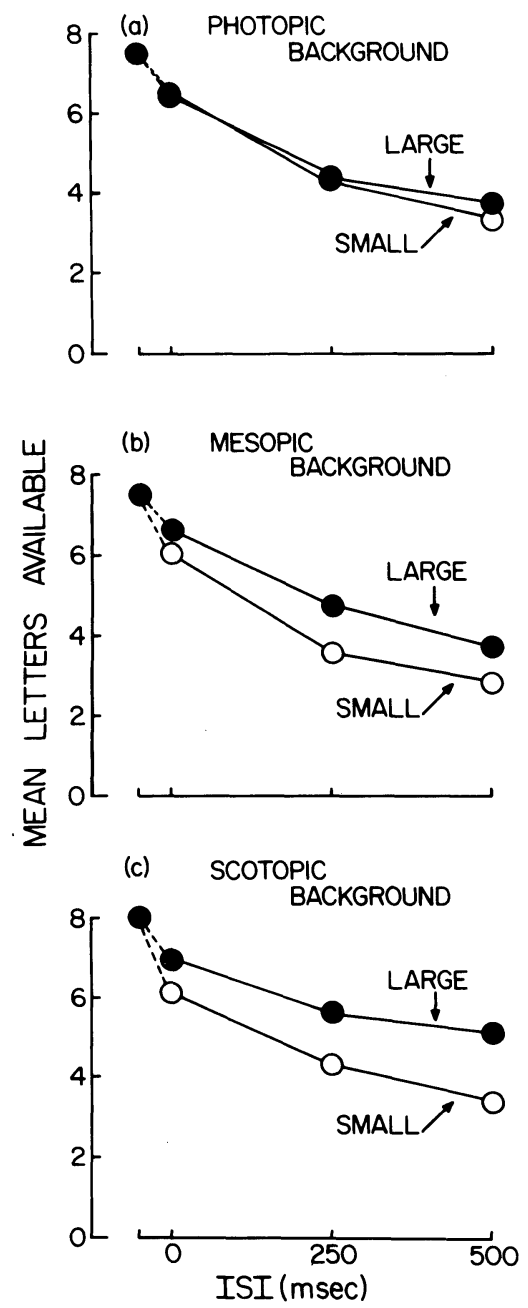

Figure 1. Mean number of letters available on a partialreport task as a function of ISI and target condition for observers run with a photopic (a), mesopic (b), and scotopic (c) background field.

functioning is greatly restricted, and persistence is essentially limited to the cone system alone. Hence, roughly equivalent functions for the two retinal eccentricities should obtain, because roughly equivalent involvement of the cone system should occur at both retinal locations. Consistent with this prediction, the results of this experiment indicate that the same number of letters were available across the two retinal locations.

The mean results for 20 observers tested under the mesopic background level are shown in Figure 1b. As with the photopic background condition presented above, these data have been subjected to a 2 (target size) $x 3$ (ISI) mixed ANOVA with repeated measures on the factor of ISI. A significant main effect of ISI is evident $[F(2,2)=21.40, p<.01]$. This again indicates that iconic storage is present at this level of background luminance. As with the photopic condition, the target size $x$ ISI interaction is not significant $[F(2,36)<1.0]$.
However, the main effect of array size is found to be significant $\left[\mathrm{F}^{\prime}(1,12)=5.23, \mathrm{p}<.05\right]$. Large targets presented away from the fovea seem to produce a greater degree of persistence than do smaller targets presented close to the fovea. These results are also believed to be compatible with the predictions of a receptor model of the icon. At the mesopic level, both the rods and the cones are functioning, and both systems are then able to contribute to visual persistence. The positive size effect in Figure 1b may then reflect a significant contribution by the rod system to task performance. Other persistence work using very different procedures has reported analogous results (e.g., Sakitt \& Long, 1979b).

Figure 1c presents the results of 20 observers tested under the scotopic background level. These data also have been subjected to a 2 (target size) $\times 3$ (ISI) mixed ANOVA with repeated measures on the factor of ISI. A significant main effect of ISI is obtained $[F(2,2)=$ $33.15, \mathrm{p}<.05$ ], reflecting the predicted decline in performance with increasing ISI. Similar to the results with the mesopic background level, the target size $\mathrm{x}$ ISI interaction fails to reach significance $[\mathrm{F}(2,36)=1.61$, $p>.05]$, and a significant main effect of target size is obtained $\left[F^{\prime}(1,15)=5.43, p<.05\right]$. Hence, under the scotopic background condition, large targets presented at the greater eccentricity produce performance superior to that produced by the small targets presented closer to the fovea. Within the retinal model, it is assumed that at this background level the rod system is operating to contribute to the persistence for the larger array size. In addition, using progressively larger letters as eccentricity increases makes a rod contribution to persistence more likely by keeping the target letters above resolution threshold for the poor-acuity rod system at each retinal eccentricity.

Finally, it should be noted that there is one pattern of results in all three portions of Figure 1 that may cause a problem for the interpretation described above. At all background levels, the degree of persistence at 0 -msec ISI is different for both target array sizes. In other words, performance was already affected at zero delay of the probe marker. Some researchers, such as Coltheart (1980), have argued that it is inappropriate to compare the persistences produced by different stimulus conditions if they also differ in their initial registration (or clarity). Consequently, any difference at later ISIs cannot be unambiguously interpreted in terms of real persistence differences. To deal with this possibility in the present work, a precue control condition was performed with a new naive observer at each background level. These observers viewed the target arrays at a -50 -msec ISI condition; that is, the probe marker preceded the array by $50 \mathrm{msec}$. This was done to determine whether performances would be equivalent for the different target sizes when no persistence whatsoever was required. As can be seen in Figure 1 for the photopic, mesopic, and scotopic levels, performance at the 
-50-msec condition does begin at, or near, optimal performance for all target sizes. It is therefore believed that obtained differences across ISI conditions are not attributable to differences in the "initial registration" of the letters, but are due to real differences in the time for decay of visual persistence. This in turn suggests that the obtained differences at 0 -msec ISI reflect some small decay in array persistence that has occurred during the brief period in which the observer is interpreting the probe's indicated direction. This may be an important methodological point, because it indicates that some persistence is involved at the 0 -msec ISI condition and that a precue condition is necessary in order to assess target clarity independently of persistence effects.

In summary, the results of this experiment are believed to be consistent with the predictions of a retinal model of visual persistence. In particular, the major point emphasized in the introduction concerning critical stimulus interactions was supported. Given such patterns of results, one must be cautious in offering any absolute statements about the character of iconic memory or even about the effect of manipulating a specific stimulus variable on iconic memory. The particular pattern of stimulus variables employed on a given task is critical.

\section{REFERENCES}

Adelson, E. H., \& Jonides, J. The psychophysics of iconic storage. Journal of Experimental Psychology: Human Perception and Performance, 1980, 6, 486-493.

Anstis, S. M. A chart demonstrating variations in acuity with retinal position. Vision Research, 1974, 14, 589-592.

Bowen, R. W., Pola, J., \& Matin, L. Visual persistence: Effects of flash luminance, duration, and energy. Vision Research, 1974, 14, 295-303.

Bowling, A., \& Lovegrove, W. Iconic memory: Fallacies persist (?). Perception \& Psychophysics, 1982, 31, 194-198.

Colthen at, M. Iconic memory and visible persistence. Perception \& Psychophysics, 1980, 27, 183-228.

Eriksen, C. W., \& Schultz, D. W. Retinal locus and acuity in visual information processing. Bulletin of the Psychonomic Society, 1977, 9, 81-84.

Eriksen, C. W., \& Schultz, D. W. Temporal factors in visual information processing: A tutorial review. In J. Requin (Ed.), Attention and performance VII. Hillsdale, N.J: Erlbaum, 1978.
HABER, R. N. The impending demise of the icon: A critique of the concept of iconic storage in visual information processing. Behavioral and Brain Sciences, 1983, 6, 1-11.

Hawkins, H. L., \& Shulman, G. L. Two definitions of persistence in visual perception. Perception \& Psychophysics, 1979, 25, 348-350.

Long, G. M. Iconic memory: A review and critique of the study of short-term visual storage. Psychological Bulletin, 1980, 88, 785-820.

Long, G. M. Persisting problems in persistence: A response to Bowling and Lovegrove. Perception \& Psychophysics, 1982, 32, 192-194.

Long, G. M., \& Benton, R. J. The case for peripheral persistence: Effects of target and background luminance on a partial-report task. Journal of Experimental Psychology: Human Perception and Performance, 1982, 8, 383-391.

Long, G. M., \& SAKitT, B. The retinal basis of iconic memory: Eriksen and Collins revisited. American Journal of Psychology, 1980, 93, 195-207. (a)

Long, G. M., \& SAkitr, B. Target duration effects on iconic memory: The confounding role of changing stimulus dimensions. Quarterly Journal of Experimental Psychology, 1980, 32, 269-285. (b)

Phillips, W. A. On the distinction between sensory storage and short-term visual memory. Perception \& Psychophysics, 1974, 16, 283-290.

SAkitt, B. Iconic memory. Psychological Review, 1976, 83, 257-276.

SakitT, B., \& Long, G. M. Relative rod and cone distributions in iconic storage. Perception \& Psychophysics, 1978, 23, $527-536$

Sakitт, B., \& Long, G. M. Cones determine subjective of fset but rods determine total persistence. Vision Research, 1979, 19, 1439-1441. (a)

Sakitt, B., \& Long, G. M. Spare the rod and spoil the icon. Journal of Experimental Psychology: Human Perception and Performance, 1979, 5, 19-30. (b)

SPERLiNG, G. The information available in brief visual presentations. Psychological Monographs, 1960, 74(11, Whole No. 498).

Whitten, D. N., \& Brown, K. T. Photopic suppression of monkey's rod receptor potential, apparently by a cone-initiated lateral inhibition. Vision Research, 1973, 13, 1629-1658. (a)

Whitten, D. N., \& Brown, K. T. Slowed decay of the monkey's cone receptor potential by intense stimuli, and protection of this effect by light adaptation. Vision Research, 1973, 13, 1659-1667. (b)

Whitten, D. N., \& Brown, K. T. The time course of late receptor potentials from monkey cones and rods. Vision Research, 1973, 13, 101-135. (c)

(Manuscript received for publication August 26, 1983.) 